\title{
Based on B/B/S Virtual Network Storage System Design and Implementation
}

\author{
Xie Chuan ${ }^{1, a}$ \\ ${ }^{1}$ Hangzhou Vocational \& Technical College, Hangzhou 310018, China \\ aXiechuan999@163.com
}

Keywords: virtual; The network; Storage; heterogeneous

\begin{abstract}
In view of the network application on the network storage requirements proposed, based on the $\mathrm{B} / \mathrm{B} / \mathrm{S}$ network storage management system concept. The system can network on each kind of heterogeneous storage server organic organization, to provide users with extensible virtual storage space. The network user can access to any network terminal the virtual storage space, save data and realize the application reads the automatic transfer.
\end{abstract}

\section{Introduction}

At present in the network environment, and a large server realized network storage function, to the web application caused the convenience. But there are also the following questions:

Users in the use of network storage service, prone to operating the fault. Log on different servers, users will need to show multiple user name and password identity authentication; User document is in not safe state, at any time there are clear by the administrator or the risk of loss; Storage space is limited, it is difficult to get effective mass file storage.

Due to the different server system mutual heterogeneous, easy to have the storage management confusion.

Often provide only file storage, unable to realize data security storage and application of automatic transfer etc. Function.

To solve these problems, establish a design based on B/B/S network storage system, the scattered, heterogeneous server storage resources for effective integration.

\section{Overall design}

According to the problems existing in the network storage, to be based on $\mathrm{B} / \mathrm{S} / \mathrm{S}$ (Browser/Server/Server) system structure of the distributed network storage system, overall design as shown in figure 1 below. Client users through the network access storage management server, through the integrated management of storage management server and the completion of the network storage space of the visit.

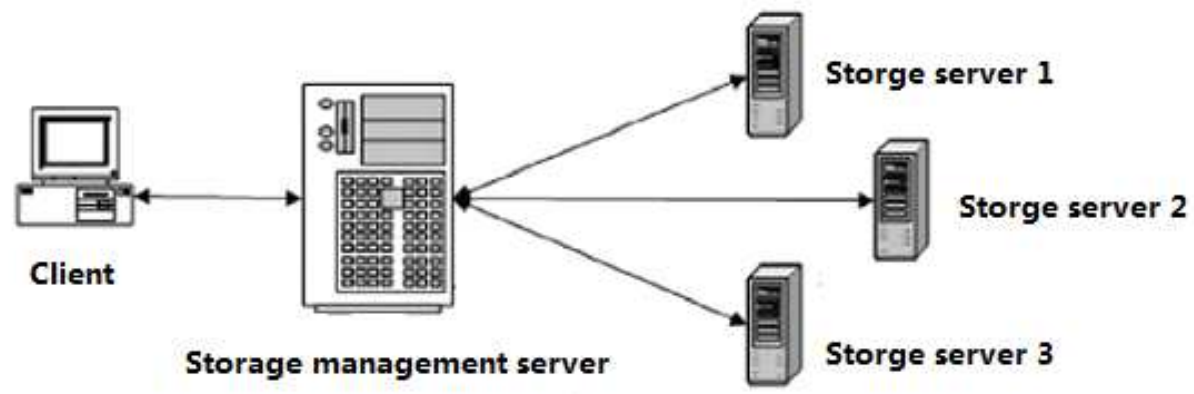

Figure 1. Overall design

The storage management Server as system Server end, mainly be responsible for the organization and management of the local store equipment and network storage Server, it mainly to complete the following features: (1) The management distributed, heterogeneous storage Server, make its are on 
the function as a complete and reliable, the large capacity of virtual storage space; (2) For the user access data provide a unified interface; (3) To the user stored in each storage server data for a fault, error correction and encryption processing, maintain the integrity of the data and confidentiality.

As a result of the $\mathrm{B} / \mathrm{S} / \mathrm{S}$ system structure, system function to most of the management of the server application implementation, and a client just through the $\mathrm{W}$ e B page or have complete access to the document agreement, reduced the client load. At the same time, all the information from management server unified management, guarantee the security of data.

\section{System structure}

Figure 2 for the network storage management system structure of the system. System including the user interface, network storage access protocol, network storage standard access interface, storage management, storage and management of the agency access storage server. The core of the system is the storage management, it also required to complete the upper users to service and of the lower storage server management both aspects of the work.

Provide service to the upper users: through the network storage access protocol for the user to provide network storage standard access interface. Through this interface, user can access to a virtual storage space, and the lower the organizational structure of the storage server to users transparent.

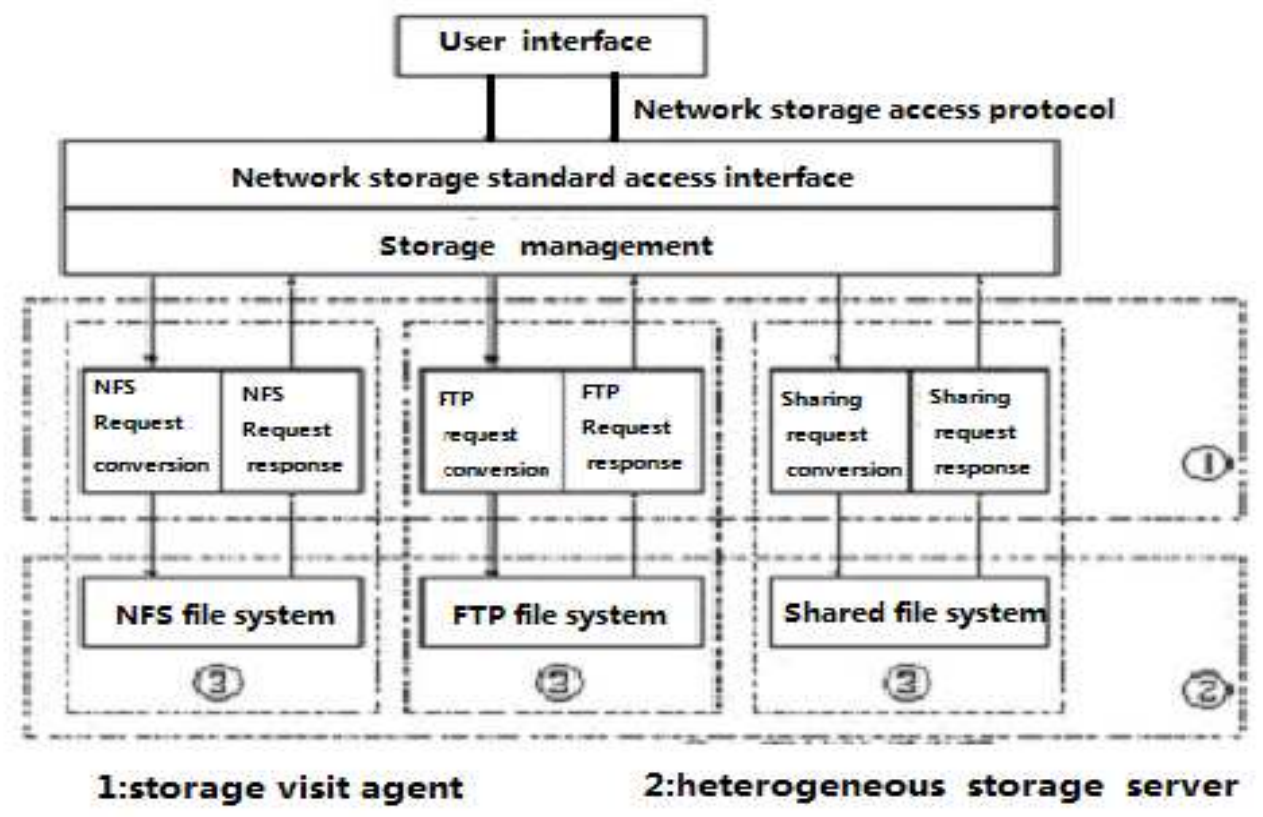

3:isomorphism storage server group management

Figure 2. System structure

Of the lower storage server management: through the memory access to the network agency distributed, heterogeneous storage server centralized management and make them form a large capacity, complete storage space, along with the interface and can be expanded to provide data security mechanism.

In the system, the user interface is mainly responsible for receiving the user to a virtual storage space access request and response to users to return to request, is the only system directly to customers information interaction between modules. The storage management module receiving user interface to send over access request, according to the user store information to the corresponding memory access directives agent, the memory access to various heterogeneous agent storage server access request of a standard.At the same time, storage management module will be collected agent of memory access each storage server to return to visit response together, through the standard access interface returned to the user interface. Memory access the main function of the 
agency is to the network of heterogeneous storage server unified management: will the heterogeneous storage server group of management, and for every "group" of the isomorphism server provide a unified interface to visit. On one hand it from a storage management module according to get orders, storage server to request data; On the other hand, will get access to the storage management module requesting to return to.

\section{System performance evaluation}

For storage system in the management system, the evaluation focused on performance and security.

Evaluate the performance of the whole system.

Because the system of management is scattered on the network, heterogeneous storage server, therefore, the performance of the system could be affected by many factors. The storage management server performance (set to $\mathrm{M}$ ) is a very important parameters, because all users of information and data must be flowing through the storage management server. If the management server processing speed and bandwidth can not meet the needs of the system, it will be bound to cause the bottleneck. In addition, the performance of the storage server (set to S) also nots allow to ignore. Because each time the user access to the operation of the storage space that is, in effect, the analytical system user access request and eventually according to user needs to storage server data access operations. In addition to the above two parameters outside, other parameters (set to $\mathrm{O}$, such as network status, etc.) also can to system performance constitute a certain effect, etc. Therefore, if use parameter P representative system performance, the $\mathrm{P}$ can be expressed as:

$$
\mathrm{P}=\mathrm{p}(\mathrm{M}, \mathrm{S}, \mathrm{O})
$$

Due to the need of the management system are distributed in storage server on the network, heterogeneous, and may be users to add to the system, the system can't active on the control and adjustment, so they in the system shows the performance may be is uneven. So, need to research on the performance of the storage server.

The performance of the storage server (S) could be affected by a variety of factors, as the own capacity, bandwidth, processing speed and reliability, etc. Can be expressed as:

$$
\mathrm{S}=\mathrm{s}\left(\mathrm{C}, \mathrm{B}, \mathrm{F}, \mathrm{R}, \mathrm{O}_{1}\right)
$$

Among them: C-storage server storage capacity; B-storage server network bandwidth; F-storage server processing speed; R-storage server reliability parameters; $\mathrm{O}_{1}$-other parameters.

(3)Storage server of the evaluation of the reliability

In the actual process, the reliability of the storage server may have significant value of the float, the impact factors basically have the following: network stability state, such as network accident interrupt, delay too big, Storage server itself performance, such as system paralyze, data failure; The attack, if the hacker attacks, infection virus; Other circumstances, such as accident without electricity, etc.

In general, can be summarized as the above factors of two types: the integrity of the data and the data network transmission speed. Among them, the data loss or failure, data infected by the virus, etc are all data integrity category; And system, accident paralyzed and delay of power belongs to the network transmission speed category situation. In addition, the collection of parameters such as time of storage server constitute the influence of reliability. Therefore, the reliability of the storage server model may be expressed as:

$$
\mathrm{R}=\mathrm{r}\left(\mathrm{I}, \mathrm{V}, \mathrm{O}_{2}\right)
$$

Among them: I-data integrity parameters; V-data transmission speed parameters; $\mathrm{O}_{2}$-other parameter design system operation process, in every visit to storage server, collect the above parameters, and combining with the actual operation of the system environment, through the mathematical model, at any time to amend the reliability value. Only reliability value in the threshold value within the storage server can be systems use. 
(4) User data safety consideration

In system design, can through the encryption, safety transmission channel technology processing, achieve the following three aspects of security guarantees:

(1)The data in storage equipment safety storage system design to be able to provide fault-tolerant services to ensure that data in storage devices not lost. That is in part storage devices, an accident cause data error or data loss, through the system of data repair mechanism, can make the destruction by accident or missing data is swift and effective recovery.

(2) Data information during transmission don't leak it contains customer information packets in the network transmission may be monitored and steal. So the system needs to provide data security transmission system, that is to ensure that the data is not lost in transmission process for the foundation. This can be realized through data encryption and other measures.

(3) Data information in a storage device not leak in the storage server system is also likely to provide other users of the system type of service. So, although the system take the identity authentication and rights management and protection measures, user data is still the risk of being stolen. In order to further defend the safety of user data, the system needs to provide user data confidential mechanism, can through the use of encryption algorithm, ensure that any information can only be its own users analytic and read correctly, so as to ensure that the user data in storage equipment privacy.

\section{Conclusion}

Through the $\mathrm{B} / \mathrm{S} / \mathrm{S}$, construct network storage management system, basically have the following features:

(1)Net users through a single login can access to a complete and reliable storage space. The system is responsible for complete the heterogeneous storage space of the unified management;

(2)User can through the Web page for the management, and simple operation, convenient;

(3)Because the system is by several network storage server integration of form, can be easily extended;

(4)System can be wrong, through test error correction provides storage for user data to ensure the safety, ensure user data on the wrong or accidental loss can recover in time.

\section{Reference}

[1] XuMeng, ZongKun. Virtual storage technology research and its application [J]. Guangxi light industry. $2010(01)$

[2] TanShengLong. Storage virtualization technology [J] micro computer application. 2010 (01)

[3] KangXiao wen, YangYingJie, DuXin. Based on virtual storage of data tolerant key technology research [J]. Computer application and research. 2009 (07)

[4] BaiXinCai, WuXueZhi, marin. Virtual storage technology and application analysis [J]. Ships electronic engineering. 2008 (5)

[5] ZhangChengFeng, XieChangSheng, LuoYiHui, LuoDongJian. Network storage unity and virtualization [J]. Computer science. 2006 (6)

[6] joash king. Based on iSCSI storage cluster in the research and design of [D]. Nanjing university of aeronautics 2010

[7] LinWei soldiers. Intelligent network storage system (INSS) storage virtualization technology research [D]. South China university of technology, 2011

[8] Chen seikei. Intelligent network storage system (INSS) storage nodes technology research [D]. South China university of technology, 2011 